\title{
Understanding the Needs of Low SES Patients with Type 2 Diabetes
}

\author{
Priscilla Barnes \\ Public Health \\ Indiana University \\ Bloomington, Indiana \\ prbarnes@indiana.edu
}

\author{
Kelly Caine \\ School of Computing \\ Clemson University \\ Clemson, SC \\ caine@clemson.edu
}

\author{
Kay Connelly \\ Informatics Computing \\ Indiana University \\ Bloomington, Indiana \\ connelly@indiana.edu
}

\author{
Katie Siek \\ Computer Science \\ University of Colorado \\ Boulder, Colorado \\ ksiek@colorado.edu
}

\begin{abstract}
This work-in-progress reports preliminary results of an interview study $(n=5)$ with low SES, rural patients with type 2 diabetes. The paper presents 3 themes and associated design suggestions relating to the high-prevalence of comorbidities, the importance of external support, and the different stages a patient may be in with respect to making lifestyle changes.
\end{abstract}

Keywords- Type 2 diabetes; diabetes self-management practices; rural communities; qualitative research; chronic disease; low SES

\section{Introduction}

Type 2 diabetes is a common chronic disease in the United States (US). In 2010, approximately 23 million people in the US population had type 2 diabetes [1]. New cases of diabetes have increased by $90 \%$ in the past decade fueled by obesity and sedentary lifestyles [2]. One goal set by the Department of Health and Human Services in its Healthy People 2020 initiative is to decrease the percent of people with diabetes who have a hemoglobin A1C level greater than 9 [3].

Over 2,000 studies (Table 1), conducted by medical and social sciences, health informatics researchers, focus on diabetes related issues. Among these studies, self-management is identified as a critical part of improving physical health and overall quality of life among people living with type 2 diabetes [4, 5]. Numerous diabetes interventions focus on intrapersonal and interpersonal factors associated with short and long term diabetes outcomes [5-7]. At the intrapersonal level, interventions primarily focused on changing knowledge, attitudes, and beliefs in an effort to influence self efficacy [56]. Knowledge and skill acquisition must take into consideration the current needs, goals, and perceived readiness to consistently implement healthy lifestyle practices [5-6]. Interpersonal factors, such as family and peer support have been instrumental in increasing external locus of control [7]. Support groups as well as individual and group coaching sessions have assisted in minimizing barriers that hinder behavior change [4-7]. Although these interventions have shown promising results in improving outcomes, disparities still persist among people with no or limited access to medical and educational interventions $[8,9]$.
Pervasive health technologies are already being used to help people make the necessary lifestyle changes to promote long-term health [10-15]. Technology has focused on recording daily activities associated with health improvement [10-12], goal setting [12], and peer support [13-15]. Individuals living with type 2 diabetes facing economic challenges may especially benefit from using health technology because it could decrease the progression of weight gain [10] encourage healthy food consumption [16], and increase physical activity [17]. Limited studies, however, have focused on this population [18-22].

Socioeconomic status (SES) is a major factor that influences who can access information and resources [8, 9]. Several type 2 diabetes interventions have been designed to address literacy and health literacy [23, 24]. Yet, more pervasive health technologies should be designed that address economic challenges. Therefore, it is important to explore determinants that may encourage or hinder persons of low SES living with diabetes to integrate technology as part of their management practices.

\section{A. Diabetes \& Chronic Disease in the HCI Literature}

Our first step towards designing technologies to help low SES people who have diabetes was to gain an understanding of the state of art knowledge about technology practices of people with diabetes. To accomplish this, we systematically examined the literature on diabetes and chronic disease that has been previously published at Pervasive Health and the ACM conference on Human Factors in Computer Human Interaction.

We queried IEEE Explore, and the ACM Digital Library for papers published at either Pervasive Health or SIGCHI using a series of advanced searches. We searched for a variety of diabetes related terms into the "title", "abstract" and "keyword" fields (Table 1). There were a total of 14 independent publications directly related to diabetes, chronic disease and/or qualitative approaches to health published in the proceedings of $\mathrm{CHI}$ and a total of 43 independent publications related to those topics presented at Pervasive Health. The first publications were in 2006 and the most recent publication was in 2012. None of these papers focused on designing for people with low SES. Designing technologies to support low-SES patients with type 2 diabetes is the focus of this paper. 


\begin{tabular}{|l|l|l|l|}
\hline Search Term & PubMed & $\begin{array}{l}\text { ACM } \\
\text { SIGCHI }\end{array}$ & $\begin{array}{l}\text { Pervasive } \\
\text { Health }\end{array}$ \\
\hline Diabetes* & 2,227 & 3 & 15 \\
\hline Diabetic & 437 & 12 & 3 \\
\hline Qualitative + Health & 26 & 2 & 9 \\
\hline Chronic Disease & 28 & 6 & 22 \\
\hline Total & 132 & 14 & 43 \\
\hline
\end{tabular}

Table 1. Papers related to diabetes and chronic disease presented at ACM SIGCHI and Pervasive Health between 2006 and 2012.

*PubMed search focus on research focused on self-management

The immediate purpose of this study is to gain an understanding of the lived experiences of low SES people in rural communities who have type 2 diabetes. The eventual goal of this work is to design pervasive health technologies to help meet their health needs. To accomplish these goals, we explore perceptions of patients receiving diabetes care at a rural community health center. Specifically, we focus on activities and practices that help participants manage their health conditions. In addition, we present design options to exemplify our findings, and advance understanding about technologies that may help low SES people with type 2 diabetes better manage their disease.

\section{Methods}

\section{A. Participant Recruitment}

Patients receiving medical care and educational counseling for type 2 diabetes at an Indiana rural community health center were recruited to participate in interviews. To participate in the study, patients were at least 18 years of age or older and were diagnosed with diabetes for at least one month or longer. The nurse practitioner and administrative staff posted flyers in the clinic and provided flyers to patients. An information sheet highlighting the purpose, benefits, and risks of study participation also accompanied the flyer. If interested in the study, patients called the researcher at the number included on the flyer and were asked questions to confirm the inclusion criteria. Face-to-face 1 hour interviews were held in a conference room at the health clinic. The research protocol was approved by Indiana University Human Subjects Institutional Review Board.

\section{B. Data collection}

Data were collected via semi-structured interviews with participants. An interview guide was developed with questions about current health condition(s) and activities or people that help participants take care of their health condition(s), daily self-management practices, challenges in managing diabetes, and use of technology in day-to-day activities. Interview questions were developed based on existing studies on the use of technology on diabetes management $[25,26]$ and previous studies on persuasive health technology conducted by coinvestigators. Questions were reviewed by the entire research team and revised to capture more targeted information and viewpoints.

\section{Data Analysis}

Interviews were transcribed by a professional transcriber and data were coded by the first author. Two levels of analysis were conducted. First, open coding was used to categorize thoughts, attitudes, and beliefs related to perceptions of diabetes self-management and the use of technology to guide activities of daily living in managing type 2 diabetes. Coding involved segmenting data into units and rearranging them into categories that facilitated insight, comparison, and clustering to a particular question or concept [27]. Transcripts were reviewed several times as a means of categorizing concepts emerging from each participant's experience. Then, transcripts were further coded based on the identification of themes through repetition, depth in contextual meaning of their experience living with diabetes, and meanings of a phenomenon shared by participants [27]. Data were organized and managed using Qualitative Solutions and Research Nonnumerical Unstructured Data Indexing (QSR NVIVO 10.0) software program. The research team read over the transcripts to ensure that nothing was omitted.

\section{Key Findings}

Five patients (mean age $=56$ ) who had been diagnosed with type 2 diabetes from 1 month to 20 years completed the interviews. All patients were White and resided in or in an adjacent county with less than 10,000 people. Highest education obtained by participants varied. Two patients were high school graduates, two patients completed some college or technical school education, and one patient was a college graduate. Two patients were employed for wages, one patient was out of work for more than one year; one patient was retired, and one patient was a homemaker. All patients reported an annual income of less than $\$ 20,000$. Patients reported having access to a computer; however, only 2 participants use the Internet. All but one participant used a mobile phone (Table 2)

\begin{tabular}{|l|l|}
\hline \multicolumn{2}{|l|}{ Demographics } \\
\hline Age (mean, range) & $56,48-64$ \\
\hline Race: white (n) & 5 \\
\hline Ethnicity: Hispanic (n) & 1 \\
\hline Gender: Female (n) & 3 \\
\hline Income (range) & $>10,000-20,000$ \\
\hline $\begin{array}{l}\text { Type 2 diabetes diagnosis (mean, } \\
\text { range) }\end{array}$ & $6.2,1$ mo-20 years \\
\hline Computer usage (n) & 5 \\
\hline Internet usage (n) & 2 \\
\hline Mobile phone usage (n) & 4 \\
\hline
\end{tabular}

Table 2. Patient demographics.

Three themes describing participants' perceptions living with type 2 diabetes were identified: (1) lived experiences in managing other health conditions in addition to diabetes; 2) external influences that promote diabetes self-management practices; and 3.) level of readiness in managing type 2 diabetes. 


\section{A. Managing co-morbidities.}

Although all participants in the study were living with type 2 diabetes, unbeknownst to the research team prior to their interviews, these individuals were also struggling to successfully manage one or more other pre-existing conditions. On average, people had 3 co-morbidities. All, but one person had high blood pressure. Headaches and back pain were common ailments among two participants while other conditions affected specific organs (e.g., liver disease, diverticulitis, thyroid) or impacted social and emotional realms of health (e.g., depression). In most cases, participants were required to take different types of medication in addition to the pill or insulin shot prescribed by the nurse practitioner in order to manage their type 2 diabetes:

I have...high blood pressure [pill], I have diabetes [pills], I've got depression [pills]. I take medicine for my thyroid. I...take a sleeping pill at nighttime. I think that's all the pills. When I had my hysterectomy my bladder fell. I can't hold my bladder so I take medicine to help kind of tighten the muscles up for my bladder. $-P 1$

Further, participants were generally overwhelmed with trying to manage all of their conditions at once, and desired to focus on a subset of their problems, with their diabetes not always being their priority.

\section{B. Responding to external influences that encourage self- management practices.}

Participants identified external factors that assist in their adoption of healthy lifestyle practices. Family members, particularly spouses/partners and siblings living nearby, attended medical appointments with participants (three of the five participants attended the medical appointment and were present at the time of the interviews and shared their point of view), ensured participants took medications, if prescribed, and reminding participants to check blood glucose levels. One of the most common references were family members' role in assisting participants make more healthful decisions about their food choices. The type of support varied and ranged from 'nagging,' to 'helpful.' Some family members were viewed as being the primary decision maker of the types of meals prepared in the household, while others were viewed as the enforcer of what not to eat. One of the female participants described the role of her son plays in helping her avoid consumption of sugary snacks:

My son is very supportive. Twice a month he'll come over and he'll stay the weekend. One day I went in [his room] and he had snacks in his suitcase and there was a Twinkie. I was like, 'oh, one isn't going to hurt me.' I reached in the bag to get it he goes, 'what are you doing?' I didn't know he was behind me and I jumped and I said, 'nothing.' He said, 'well you do know that Twinkie is not for you, right?' I said, 'yes.' - PI

Friends, particularly those who also have diabetes, were sought for suggestions and advice about food choices or being a 'catalyst' for change by spurring participants to discontinue practices that were viewed as not conducive to maintaining adequate blood glucose levels. A participant living with diabetes for 10 years describes how a friend who underwent surgery to remove his leg reminded her of the consequences of improper control of blood glucose levels:

My friend...lost his leg because he didn't do what they told him to do. He might take his medicine, he might not, you know,... and he was worst than me... he had somehow or another got a sore on his ankle and he tried to take care of it and he didn't. And then that sore...he got gangrene in it and they... had to take off his left foot. So I mean that was a motivator for me.... really bad. I said 'dear Lord, I'm living the same way he is...' - Pl

The healthcare provider was seen as a key factor in encouraging self-management practices. Participants looked to the healthcare provider to provide reliable information, communicate information in an understandable manner, and show concern as well as empathy about progress patients make in their care. Participants recognized that challenges may occur in their change towards consistent self management, but most participants appreciate the patience of their provider even when such progress towards healthful change is slow. In addition to the importance of having a health care provider who is knowledgeable about diabetes pathology, the effect medication has on treating their condition and being able to thoroughly explain the impact of lifestyle practices on improving diabetes, one participant's significant other, who attended the interview, expressed her appreciation that the nurse practitioner holds him accountable for his action:

She doesn't accept excuses. When he said something about I don't exercise because I hurt, she said well you know, basically you need to fix that because that's not an excuse not to exercise. You've got to do something so that you can. Or if he said well, the diet. Whatever. She put it out there and said this what you've got to do. To protect your health, you've got to do it. And I liked that. - P4

\section{Recognizing different levels of readiness are involved in diabetes self-management.}

This study comprised older adults spanning from a few month to several years of living with diabetes. Participants were either 1) thinking about and/or 2) preparing to address a lifestyle practice in order to control their blood glucose levels or another condition. For example, all participants mentioned the importance of proper nutrition including eating breakfast, consuming at least every 3-4 hours, maintaining proper portion sizes, and reducing the amount of sugary foods (e.g., desserts, white bread) and beverages (soda). Two of the five participants (diagnosed with diabetes less than 6 months) were working with the diabetes educator in order to improve their nutritional habits. Both individuals were willing to work with a health professional, however, one shared he thought that the healthcare provider was lying about his diagnosis because he did not have a previous family history of the disease; however, he appeared to be at the stage where he is accepting he had 
diabetes. Two participants (one person living with diabetes for 10 and the other person 20 years) described ongoing challenges of maintaining healthy food practices, but discussed the importance of being aware of social and environmental settings as well as setting small achievable goals especially when they experienced "ruts."

I'll limit myself to one pop a day...if I go to two or three people's houses...I'm drinking all that pop. I drink my tea with no sugar in it now because of that. That's the big thing because man I'll tell you...I'd drink them pops in a heartbeat if I didn't use my head. Since she's putting me on [insulin] it's scared me.... because it's so easy for me to go back. -P1

Weight loss is my goal. I was there for a long time like 190. For the last year, I've been at 185. I'm kind of stuck. I mean I have been trying here more lately because anytime I'd get hungry I'd eat. I didn't care what time it was, day or night. Now I'm eating four to five meals a day. -P3

One participant (living with diabetes for 1 year) recognized that she was not making strides to improve her nutritional practices, but was more interested in quitting smoking:

It's been about a year ago [since seeing the diabetes educator].Somebody just needs to take a club and hit me in the head. I know I need to quit smoking. I'm wanting to eat, but it's hard. I'm trying to quit [smoking]. - P5

\section{Discussion}

Many of the needs of low-SES patients with diabetes are similar to that of their high-SES counterparts. Education, sensemaking, personalized tacking and feedback are all goals that have been studied previously [CHAP] and also make sense for our target population. The preliminary findings of our interviews, however, uncovered additional design considerations that are particularly important for this population:

Design Consideration \#1: Technology should not only assist patients with managing their type 2 diabetes, but should incorporate information about how other conditions they are living with impact their health. Prior design work has focused on a single condition (e.g. type 2 diabetes), and has not embedded co-morbidities into the core of a design [2830]. Technological devices, such as smart phones or wireless pedometers, have served as an accountability tool to assist individuals monitor blood glucose levels and activities of daily living (e.g., engaging in physical activity, eating at least three to four meals a day, taking medications as prescribed) Unfortunately, individuals who have limited financial resources and live in areas where these devices are not readily available are not able to benefit, In addition, most devices have textual based interfaces that assumes the individuals have high literacy skills. This notion again makes technological health interventions inaccessible to many low SES individuals. Few technology researchers have focused on this population [20-21]; particularly in understanding what drives adoption of these resources. In this study, all but one person had access to at least one device (a cell phone), but they were mainly unaware of the resources that could be used to help manage their health conditions outside of their primary care provider. Creating a system interface that is cost effetive and has the potential to 'digitally connect' individuals with limited resources to health information can build their self efficacy as well as empower them to take charge of their own health.

Design Consideration \#2: Technology must be flexible by taking into consideration a person's level of readiness to make a particular change in their lifestyle. At every level, it is important to emphasize concrete steps the patient can take, without overwhelming them. When combined with Design Consideration \#1, this means that technology may need to adapt to focus on different health conditions at different times, as addressing them all at once may be too much. Existing literature takes into account the stages-of-change model for how to best communicate and encourage patients improve their personal health status [30]. This study highlights that patients with multiple co-morbidities may be in different stages for many different behavior changes. Previous approaches use disease-focused interventions rather than human-centered interventions that take into account the current experiences that individuals with multiple chronic conditions face on a daily basis. Also, interventions fail to reflect the stage-based process of health behavior change. To address these gaps, we are currently designing technologies that actively engage patients in progressing through stages of behavior change

Design Consideration \#3: Technology should include a component that involves family and friends who are directly involved in assisting a person with self-management practices. Similar to [28], our work recognizes the critical importance of both a patient's social network and health provider network in supporting and negotiating chronic disease management practices. Peer support offered through mobile phone has been found to be useful in enhancing diabetes self practices. This type of support may be of greater importance for persons of low SES residing in rural areas since access to educational resources and health services are major issues of concern. Thus, it is imperative to create technologies that facilitate conversations between a patient, their loved ones, and health providers in order to create an environment based on encouragement and accountability.

\section{v. Conclusions}

Our preliminary work provides insight into the design of technologies to help low-SES patients with type 2 diabetes. Gathering contextual information that type 2 diabetes patients face and live with on a daily basis is critical in creating devices that acknowledge successes as well as address ongoing challenges. Understanding intrapersonal factors, such as beliefs and knowledge about diabetes and existing comorbidities, may influence self efficacy and the adoption of self management practices. The vision of this research is to create a design that addresses intrapersonal, interpersonal, 
community, and societal factors (a socio-ecological perspective) that shepherds a person through a social supportive process to achieve specific, measurable, achievable, realistic, and timely goals.

To that end, our future work will explore the development of specific self management and design strategies that encourage progression from 'contemplating', to 'preparing', to 'taking' action" for a variety of relevant health behaviors, including improving blood glucose levels. Because health is complex and is not solely based on individual behavior, environmental and community context will also need to be examined. Finally, our participants were from rural settings, and we plan to continue our work with low-SES urban patients as well.

\section{Acknowledgements}

We thank our participants and the nurse practitioner, Linda Wells Freiberger, who assisted us in recruitment. This work was supported in part by a grant from the Department of Applied Health Science, Indiana University School of Public Health, the Center for Law, Ethics, and Applied Research in Health Informatics, and the School of Informatics and Computing at Indiana University.

\section{References}

[1] Centers for Disease Control and Prevention. National Diabetes Fact Sheet. 2011. Retrieved http://www.cdc.gov/diabetes/pubs/estimates11.htm\#1

[2] American Diabetes Association. New US diabetes rate up to 90 percent in past decade. 2006. Retrieved at: http://forecast.diabetes.org/news/new-us-diabetes-rate-90-percent-pastdecade

[3] Department of Health and Human Services. Healthy People 2020. 2010. Retrieved at: http://www.healthypeople.gov/2020

[4] Funnel M, Brown T, Childs B, Haas L, Hosey G, Jensen B et al. National standards for diabetes self management education. Diabetes Care. 2008. 32(1): S87-S94.

[5] Rotheram-Borus M, Ingram B, Swendeman D, Lee A. Adoption of self management interventions for prevention and care. Primary Care. 2012. 39(4): 649-660

[6] Renders C, Valk G, Griffin S, Wagner E, Jacquest ThM E, Assendelft $\mathrm{W}$. Interventions to improve the management of diabetes in primary care, outpatient, and community settings: A systematic review. Diabetes Care. 2001. 24(10): 1821-1833.

[7] Matthew R, Gucciardi E, De Melo , Barata P. Self-management experiences among men and women with type 2 diabetes mellitus: a qualitative analysis. BMC Family Practice. 2012. 19(12): $122 .$.

[8] Adler, N.E. and K. Newman, Socioeconomic Disparities In Health: Pathways And Policies. Health Affairs, 2002. 21(2): p. 60-76.

[9] Glazier R, Bajcar J, Kennie N, Wilson K. A systematic review of interventions to improve diabetes care in socially disadvantaged populations. Diabaetes Care. 2006. 29(7): 1675-1688.

[10] Nachman, L., Baxi, A., Bhattacharya, S., Darera V., Deshpande , P., Kodalapura, N., et al. Jog Falls: A pervasive healthcare platform for diabetes management. Pervasive Computing. 2010. 6030: 94-111.

[11] El-Gayar O, Timsina P, Nawar N. Eid W. Mobile applications for diabetes self-management: status and potential. J Diabetes Sci Technol. 2013. 7(1): 247-262.

[12] Mulvaney S, Ritterband M, Bosslet L. Mobile intervention design in diabetes: review and recommendations. Curr Diab Report. 2011. 11(6): 486-493.
[13] Willard-Grace R, Devore D, Chen E, Hessler D, Bodenheimer T, Thom D. The effectiveness of medical assistant health coaching for patients with uncontrolled diabetes, hypertension, and hyperlipidemia: protool for a randomized controlled trial and baseline characteristics of the study population. BMC Family Practice. 2013. 23:14-27.

[14] Jethwani K, Ling E, Mohammed M, Myint U, Pelletier A, Kvedar J. Diabetes connect: an evaluation of patient adoption and engagement in a web-based remote glucose monitoring program. Joruanl Diabetes Scientific Technology. 2012. 6(6): 1328-1326.

[15] Rotheram-Borus M, Tomlinson M, Gwegwe M, Comulada W, Kaufman N, Keim M. Diabetes buddies: peer support through a mobile phone buddy system. Diabetes Educator. 2012 38(3): 357-365.

[16] Connelly, K., Siek, K.A., Chaudry, B., Jones, J., Astroth, K., and Welch, J. An Offline Mobile Nutrition. Monitoring Intervention for Varying Literacy Patients Receiving Hemodialysis: A Pilot Study Examining Usage and Usability. Journal of the American Medical Informatics Association, 2012. 19:705-712.

[17] Clark, D., Chrysler, L., Perkins, A., et al: Screening, referral, and participation in a weight management program implemented in five CHCs. J Health Care Poor Underserved 2010; 21:617-628.

[18] Siek, K.A., J.S. LaMarche, and J. Maitland. Bridging the information gap: collaborative technology design with low-income at-risk families to engender healthy behaviors. in Proceedings of the 21st Annual Conference of the Australian Computer-Human Interaction Special Interest Group: Design: Open 24/7 2009. Melbourne, Australia: ACM.

[19] Maitland, J., Siek, K.A., and Chalmers, M., Persuasion not Required: Obstacles Faced by Low-Income Caregivers to Improve Dietary Behaviour. 3rd International Conference on Pervasive Computing Technologies for Healthcare, 2009.

[20] Maitland, J. and Siek, KA. Technological approaches to promoting physical activity. in Proceedings of the 21st Annual Conference of the Australian Computer-Human Interaction Special Interest Group: Design: Open 24/7 2009. Melbourne, Australia: ACM.

[21] Siek, K.A. and J. Maitland, Studying the place of Technology to Lower Financial Barriers for Dietary Change. Methods of Information in Medicine, 2009. 49(1): p. 74-80.

[22] Grimes, A., et al., EatWell: sharing nutrition-related memories in a lowincome community. CSCW '08: Proceedings of the ACM 2008 conference on Computer supported cooperative work, 2008: p. 8796.McMahon GT, Gomes HE, Hickson Hohne S, Hu TM, Levine BA, Conlin PR: Webbased care management in patients with poorly controlled diabetes. Diabetes Care. 2005. 28:1624-1629.

[23] Saha, S., Improving literacy as a means to reducing health disparities. Journal of General Internal Medicine, 2006. 21(8): p. 893-895.

[24] Pignone, M., et al., Interventions to Improve Health Outcomes for Patients with Low Literacy: A systematic review. Journal of General Internal Medicine 2005. 20(2): p. 182-192.

[25] Carlson, B., Neal D, Magwood, G., Jenkins C, King M., Hossler, C. A community-based participatory health information needs assessment to help eliminate diabetes information disparities. Health Promotion Practice. 2006. 7(3): 213S-222S.

[26] Lena Mamykina, Elizabeth D. Mynatt, and David R. Kaufman. 2006. Investigating health management practices of individuals with diabetes. In Proceedings of the SIGCHI Conference on Human Factors in Computing Systems (CHI '06), Rebecca Grinter, Thomas Rodden, Paul Aoki, Ed Cutrell, Robin Jeffries, and Gary Olson (Eds.). ACM, New York, NY, USA, 927-936. http://doi.acm.org/10.1145/1124772.1124910

[27] Saldana J. The Coding Manual for Qualitative Researchers. Jossey-Bass. Thousand Oaks, CA. 2009.

[28] Maitland J. Towards negotiation as a framework for health promoting technology. ACM SIGHIT. 2011. 1(1): 10-19.

[29] Toscos, T., Connelly, K. Using Behavior Modification Theory to Understand and Guide Technological Interventions In Bill Aspray and Barb Hayes, editors, Health Informatics: A Patient-Centered Approach to Diabetes, pp. 295-326, MIT Press, October 2010.

[30] Kerr E, Heisler M Krein S, Kabeto M, Langa KM, Weir D, Piette. Beyond comoridity counts: how do comorbidity type and severity influence diabetes patiet treatment priorites and self-management? J Gen Intern Med. 2007. 22(12); 1635-1640. 